\title{
Tratamiento individualizado de la deshidratación hipernatrémica en el recién nacido
}

\author{
Individualized treatment of hypernatremic dehydration in \\ newborn infant
}

Carlos López-Candiani

\section{ANTECEDENTES}

La hipernatremia (sodio sérico $145 \mathrm{mEq} / \mathrm{L}$ ) es un desequilibrio que, cuando es grave $(160 \mathrm{mEq} / \mathrm{L})$, puede causar alteraciones vasculares y neurológicas agudas, dejar secuelas graves o causar la muerte en recién nacidos. ${ }^{1-4}$ Se han descrito: trombosis del seno sagital superior, ${ }^{5-6}$ aórtica ${ }^{7-8}$ mesentérica, renal, iliaca, femoral, poplítea ${ }^{7}$ y arteria retinal; ${ }^{9}$ hemorragia parenquimatosa, ${ }^{10}$ gangrena distal,, $11-12$ enterocolitis necrosante, ${ }^{13}$ hipertensión arterial pulmonar e insuficiencia cardiaca derecha. ${ }^{14} \mathrm{La}$ patogénesis de las lesiones cerebrales se describió hace más de medio siglo, ${ }^{15}$ muchas secundarias a una corrección inadecuada. Oddie ${ }^{16}$ informa una incidencia de 7 x cada 100,000 nacidos vivos. Se trata, en sí, de un trastorno de la concentración relativa de sodio en el espacio extracelular, y no necesariamente de la cantidad de sodio corporal total que puede estar elevada o ser normal; puede asociarse con volumen de agua corporal normal, alto o bajo.

La hipovolemia puede ser secundaria a un aporte insuficiente de agua, a pérdidas incrementadas o ambas. ${ }^{17}$ En la actualidad, una de las causas más frecuentes de hipernatremia es la deshidratación asociada con amamantamiento exclusivo, pero ineficaz. 2,16,18-22 Lavagno encontró 115 publicaciones de esta relación en una revisión sistemática publicada en 2016. ${ }^{23}$ Las complicaciones extracerebrales de la deshidratación hipernatrémica incluyen a la insuficiencia renal aguda, elevación de enzimas hepáticas, acidosis metabólica, hipoglucemia o hiperglucemia y coagulación intravascular diseminada, entre otras. ${ }^{24}$

El objetivo de este criterio pediátrico es aportar un método de corrección de sodio y agua individualizado para cada grado de deshidratación y concentración sérica de sodio que evite las complicaciones neurológicas secundarias a la corrección rápida.
Neonatólogo, subdirector de Medicina Crítica.

Instituto Nacional de Pediatría, Ciudad de México

Recibido: 29 de noviembre 2018

Aceptado: 12 de febrero 2019

Correspondencia

Carlos López Candiani

mensaje55@hotmail.com

Este artículo debe citarse como López-Candiani C. Tratamiento individualizado de la deshidratación hipernatrémica en el recién nacido. Acta Pediatr Mex. 2019;40(2):99-106. 


\section{Fisiopatología}

El aumento en la concentración de sodio sérico implica incremento de la osmolaridad; es decir, un estado hipertónico. Por efecto de la ósmosis hay un movimiento de agua del espacio intracelular al extracelular hasta que se iguala la osmolaridad dentro y fuera de la célula; esto Ileva a una pérdida del líquido intracelular.25,26

El sistema nervioso central tiene la capacidad de captar electrolitos (sodio, potasio, cloro) en forma inmediata (menos de una hora) y de sintetizar aminoácidos y solutos orgánicos osmoprotectores en forma mediata. Estos aminoácidos incluyen: glutamato, glutamina, fosfocreatina y taurina. Los solutos orgánicos (osmoles idiógenos) incluyen: mioinositol, glicerofosforilcolina y betaína. Estos osmoles idiógenos mantienen el volumen cerebral y protegen a las proteínas intracelulares durante periodos prolongados de estado hiperosmolar sérico y son una respuesta diferencial en el sistema nervioso central. ${ }^{25}$

En el trascurso de una semana el cerebro recupera $98 \%$ del agua perdida. ${ }^{26} \mathrm{Si}$ en un estado hipernatrémico (hiperosmolar) se hace una corrección rápida, la mayor parte de las células del cuerpo se rehidratarán de forma adecuada; sin embargo, en el cerebro que ha mantenido su volumen intracelular entrará líquido, igualmente rápido, desde el espacio extracelular que llevará a la neurona a edema. En el curso de la rehidratación se han descrito convulsiones y edema cerebral; ${ }^{27-28}$ la patogénesis se describió hace más de 50 años. ${ }^{29}$ Para el tratamiento de la hipernatremia se recomienda la corrección lenta, a una velocidad no mayor de $0.5 \mathrm{mEq} / \mathrm{L} / \mathrm{h}$ de sodio sérico, ${ }^{30-31}$ para evitar edema cerebral. Previamente documentamos una evolución favorable con descensos de sodio menores a $0.6 \mathrm{mEq} / \mathrm{L} / \mathrm{h} .{ }^{32}$

\section{Tratamiento}

Cada libro de texto y artículo que trata el tema ofrece una fórmula diferente para la corrección del sodio. La mayor parte son fórmulas fijas (determinadas en $\mathrm{mL} / \mathrm{kg} /$ día de agua libre o concentración fija de sodio en las soluciones). El estado osmolar será diferente conforme el neonato pierde mayor proporción de agua y se eleva la concentración de sodio y, por ende, la osmolaridad sérica. El método aquí expuesto es resultado de una revisión extensa de la bibliografía, modificación de fórmulas y aplicación en la clínica durante una década.

Harding ${ }^{33}$ menciona que, si no hay choque, la rehidratación puede efectuarse por vía enteral, con leche humana extraída o un sustituto. BenShalom y su grupo ${ }^{30}$ recurrieron a la vía enteral aproximadamente 10 horas después de iniciar la rehidratación parenteral, con una mediana de sodio sérico de $156 \mathrm{mEq} / \mathrm{L}$. En un estudio previo de nuestro grupo acerca de factores de riesgo de desenlace adverso en neonatos con hipernatremia ${ }^{32}$ se observó, entre los neonatos en quienes la corrección se efectuó por vía oral, que una concentración de sodio de 160 $\mathrm{mEq} / \mathrm{L}$ correspondió al percentil 75 de los que tuvieron un desenlace adecuado y la misma cifra correspondió al percentil 25 de los que tuvieron mala evolución. Con base en esto se decidió que en todos los neonatos con sodio sérico menor de $160 \mathrm{mEq} / \mathrm{L}$, y en quienes las condiciones del abdomen son adecuadas y no hay alguna contraindicación para la ingesta oral, ésta es la vía de corrección. Se prefiere la leche humana (fórmula de inicio en su ausencia) porque garantiza un volumen adecuado que satisface los requerimientos y recupera el déficit.

Debe tenerse cuidado de no usar agua estéril oral porque se ha asociado con enterocolitis y perforación intestinal en neonatos con hipernatremia. ${ }^{34} \mathrm{En}$ todos los neonatos con sodio sérico de $160 \mathrm{mEq} / \mathrm{L}$ o más, o en quienes haya contraindicación para la vía oral, se hará un cálculo de soluciones parenterales. El cálculo siguiente es válido para el neonato que aún mantiene diuresis 
López Candiani C. Tratamiento de hipernatremia neonatal

(aunque haya oliguria). Para el neonato en anuria se requiere un cálculo ligeramente diferente que presentaremos próximamente.

Los objetivos del tratamiento son:

1. Mantener el volumen intravascular: tratar el choque si lo hay.

2. Recuperar el líquido perdido.

3. Restablecer el equilibrio entre compartimientos: deshidratación celular.

4. Establecer una tasa de disminución de sodio que no exceda $0.5 \mathrm{mEq} / \mathrm{L} / \mathrm{h}$.

5. Proteger al cerebro.

Si a la llegada al hospital el neonato acude en estado de choque, deberá tratarse con una carga rápida a razón de $10 \mathrm{~mL} / \mathrm{kg}$; por lo general en ese momento no se conoce que se trata de un estado hiperosmolar, por lo que regularmente se indica solución fisiológica que contiene $154 \mathrm{mEq} / \mathrm{L}$ de sodio. Si se conoce la concentración sérica de sodio (ante una eventual segunda carga rápida) se indicará una solución con concentración de sodio $20 \mathrm{mEq} / \mathrm{L}$ menor que la concentración sérica, a $10 \mathrm{~mL} / \mathrm{kg}$. Cuando el neonato ya no tenga manifestaciones de choque se procede a planear el tratamiento.

Si el objetivo es disminuir el sodio a $0.5 \mathrm{mEq} / \mathrm{L} / \mathrm{h}$, al hacer los cálculos para 24 horas, lo ideal será el descenso de $12 \mathrm{mEq} / \mathrm{L}$ de sodio. Si el neonato tiene, inicialmente, una concentración de sodio extremadamente alta, se planeará la corrección para tantos días como permita un descenso de 12 $\mathrm{mEq} / \mathrm{L} /$ día. Si acude con $180 \mathrm{mEq} / \mathrm{L}$, el primer día se planeará la disminución de 180 a 168 mEq/L, el segundo de 168 a $156 \mathrm{mEq} / \mathrm{L}$ y el tercer día de 156 a $144 \mathrm{mEq} / \mathrm{L}$.

El ABC del tratamiento de la deshidratación hipernatrémica incluye:
A. Agua libre necesaria para diluir el sodio $12 \mathrm{mEq} / \mathrm{L}$ en 24 horas.
B. Volumen de líquido a pasar en las siguien- tes $24 \mathrm{~h}$.
C. Concentración de sodio en las soluciones.

\section{A. Agua libre}

El concepto de agua libre de una solución considera el agua disponible para movimiento osmótico a través de una membrana semipermeable. Por ejemplo: si en el espacio extravascular hay una concentración de sodio de $150 \mathrm{mEq} / \mathrm{L}$ y se infunde una solución con la misma concentración, estará osmóticamente equilibrada y no habrá movimiento a través de la membrana celular. Sin embargo, si se infunde una solución con concentración de sodio de $75 \mathrm{mEq} / \mathrm{L}, 50 \%$ del líquido será osmóticamente activo y el otro $50 \%$ estará libre para moverse a través de la membrana celular. Si se infunde en una cantidad de $100 \mathrm{~mL}$, entonces $50 \mathrm{~mL}$ serán agua libre. Más aún, si se usa una solución con sodio a $50 \mathrm{mEq} / \mathrm{L}$, entonces una tercera parte ejercerá presión osmótica y dos terceras partes estarán disponibles para moverse a través de la membrana semipermeable. Si se infunden $100 \mathrm{~mL}, 33 \mathrm{~mL}$ quedarán en el espacio extracelular y $66 \mathrm{~mL}$ se moverán al interior de las células. Al aplicar la fórmula de agua libre, se estará calculando cuánta agua (pura) es necesaria para diluir el sodio sérico 12 $\mathrm{mEq} / \mathrm{L}$ y se infundirá en las siguientes $24 \mathrm{~h}$. Al modificar la fórmula de Molteni ${ }^{35}$ se expresa de la siguiente manera:

Fórmula 1. Agua libre $(\mathrm{mL})$.

$$
\frac{12 * \text { peso }(\text { gramos }) * \begin{array}{c}
\text { proporción } \\
\text { de agua corporal }
\end{array}}{\text { Sodio sérico actual }}
$$

12 significa los $\mathrm{mEq} / \mathrm{L}$ de sodio que deseamos reducir en las siguientes $24 \mathrm{~h}(0.5 \mathrm{mEq} / \mathrm{L}$ por hora). Se utiliza el peso antes de la deshidrata- 
ción expresado en gramos; en la práctica se usa el peso de nacimiento para neonatos que acuden a partir de los 7 días de vida; para neonatos de menor edad se calcula el descenso fisiológico hasta $10 \%$ y ese será el peso para anotar. La proporción de agua corporal en el neonato de término sano es de 0.75 ; sin embargo, el neonato deshidratado pierde agua y debe restarse la proporción de peso que perdido al 0.75 habitual.

Es muy difícil saber exactamente cuánta agua ha perdido el paciente, pero en el caso del recién nacido hay al menos un peso fidedigno al nacer. De acuerdo con von Dommelen ${ }^{36}$ la mediana de pacientes nacidos a término recupera el peso de nacimiento a los 8 días. Por ejemplo, un neonato que acude a los 10 días con pérdida de peso del $20 \%$ respecto al nacimiento se asume una deshidratación del mismo porcentaje. En este ejemplo, la proporción de agua corporal a anotar en la fórmula 1, será de: $0.75-0.20=0.55$.

Para facilitar la aplicación de las fórmulas se usará el siguiente ejemplo: el paciente $\mathrm{N}$ con peso al nacer de 3000 g acude al día 8 de vida con 2400 g y Na sérico de $170 \mathrm{mEq} / \mathrm{L}$. La pérdida de peso corresponde a 20\% (proporción de 0.2 ). La proporción de agua corporal en ese momento es de 0.75-0.2 =0.55; luego entonces:

Sustitución de fórmula 1. Agua libre $(\mathrm{mL})$ :

$$
\frac{12 * 3000 * 0.55}{170}=116.47
$$

La interpretación del resultado es que el bebé $\mathrm{N}$ requiere $116.5 \mathrm{~mL}$ de agua libre (pura) para diluir el sodio de 170 a 158, que se pasará en las siguientes 24 h; más adelante se explica cómo.

\section{B. Volumen de líquido para 24 h}

Para calcular el volumen de líquido a pasar en las siguientes $24 \mathrm{~h}$ deben considerarse tres aspectos:
1. Déficit previo (volumen de líquido perdido por deshidratación).

2. Líquidos de requerimiento para las siguientes $24 \mathrm{~h}$.

3. Cargas rápidas administradas al ingreso.

Así como no se desea la corrección rápida de sodio, tampoco se quiere la corrección rápida de agua, porque permitiría mayor movilización a través de la membrana celular.

El déficit previo se calcula como la diferencia entre los pesos (nacimiento y actual); en el caso del paciente $\mathrm{N}$ se calcula de la siguiente manera: $3000-2400 \mathrm{~g}=600 \mathrm{~g}$; con esto se asume que el déficit es de $600 \mathrm{~mL}$. Como la corrección de sodio se realizará 2 días (170 a $158 \mathrm{mEq} / \mathrm{L}$ el primer día y a $146 \mathrm{mEq} / \mathrm{L}$ el segundo), entonces se programa la recuperación del déficit a $48 \mathrm{~h}$. El déficit de $600 \mathrm{~mL}$ se divide entre 2 días y el primero se infunden $300 \mathrm{~mL}$ como déficit.

Los líquidos de requerimiento se calculan acordes con la edad del neonato. En esta unidad se calculan a $150 \mathrm{~mL} / \mathrm{kg} /$ día para la edad del paciente $\mathrm{N}$, que multiplicados por $3 \mathrm{~kg}$ (peso inicial), resulta en un volumen de requerimiento de $450 \mathrm{~mL}$. En caso de que el paciente tenga menos de 7 días, se calculan de acuerdo con los requerimientos diarios; en esta unidad se inicia con $80 \mathrm{~mL} / \mathrm{kg} /$ día el primer día y luego $10 \mathrm{~mL} /$ $\mathrm{kg} /$ día cada día, de tal forma que a la semana de vida ya se calculan $150 \mathrm{~mL} / \mathrm{kg} /$ día.

Se suma el déficit a corregir (300 mL) más los requerimientos $(450 \mathrm{~mL})$ y se obtiene un volumen de $750 \mathbf{~ m l ~ p a r a ~} 24 \mathrm{~h}$.

En caso de que haya sido necesario pasar una o más cargas rápidas para tratar un eventual estado de choque, el volumen total administrado en las cargas debe restarse al cálculo de volumen de líquido para $24 \mathrm{~h}$ en este momento. En este caso no se resta el volumen de cargas rápidas asumiendo que no se infundieron. 
López Candiani C. Tratamiento de hipernatremia neonatal

\section{Concentración de sodio}

Para el paciente $\mathrm{N}$ se infundirán $750 \mathrm{~mL}$ para $24 \mathrm{~h}$, de los que $116.5 \mathrm{~mL}$ serán de agua libre, que representa $15.5 \%$ del total de volumen a infundir. Para obtener la concentración de sodio de las soluciones, se resta al $100 \%$ de los líquidos el volumen que corresponde al agua libre: $100 \%-15.5 \%=84.5 \%$; esta cifra representa la proporción de líquido que debe ser osmóticamente activo para la concentración de sodio que tiene el paciente actualmente; entonces se aplica la fórmula siguiente:

Fórmula 2. Concentración de sodio en las soluciones $(\mathrm{mEq} / \mathrm{L})$ :

$$
\frac{\text { Na sérico actual * }\left(\begin{array}{c}
100-\text { porcentaje } \\
\text { de agua libre }
\end{array}\right)}{100}
$$

Sustituyendo:

$$
\frac{170 *(100-15.5)}{100}=143.6 \mathrm{mEq} / \mathrm{L}
$$

Los $750 \mathrm{~mL}$ que se infundirán al paciente $\mathrm{N}$ deberán tener una concentración de sodio de $143.6 \mathrm{mEq} / \mathrm{L}$; de esta forma recibirá $116.5 \mathrm{~mL}$ de agua libre y el sodio descenderá $12 \mathrm{mEq} / \mathrm{L}$ en las siguientes $24 \mathrm{~h}$.

Por regla de proporciones, si se desea preparar soluciones con Na a $143.6 \mathrm{mEq} / \mathrm{L}$, para $750 \mathrm{~mL}$ se requieren $107.7 \mathrm{mEq}$ de sodio. Se usa sodio concentrado al $17.7 \%$ para preparar las soluciones, considerando que $1 \mathrm{~mL}$ de solución tiene 3 $\mathrm{mEq}$ de sodio, por lo que se utilizarán $35.9 \mathrm{~mL}$ para obtener los $107.7 \mathrm{mEq}$ de sodio requeridos para la solución de 24 horas.

\section{Agua libre de una solución}

Para comprobar que la solución con sodio a $143.6 \mathrm{mEq} / \mathrm{L}$ tiene el agua libre necesaria se aplica la siguiente fórmula:

Fórmula 3. Proporción de agua libre de una solución:

1 - (Na en solución/ Na actual)

Sustituyendo:

$1-(143.6 / 170)=0.155$

La proporción de agua libre de esta solución para el sodio sérico en el paciente $\mathrm{N}$ es de 0.155 ; es decir, $15.5 \%$ de la solución será agua libre y el $84.5 \%$ será líquido osmolarmente activo.

Para el paciente $\mathrm{N}$ se desea administrar una tasa de glucosa a $5 \mathrm{mg} / \mathrm{kg} / \mathrm{min}$. Se debe calcular con base en el peso previo a la deshidratación (en el ejemplo se usó el peso al nacer de $3000 \mathrm{~g}$ ). Entonces se multiplican $5 \mathrm{mg} * 3 \mathrm{~kg} * 1440 \mathrm{~min}$ del día, y se obtienen: $21,600 \mathrm{mg}$ de glucosa (21.6 g). Se usa dextrosa al 50\% (50 g/ $100 \mathrm{~mL}$ ) para obtener los gramos de carbohidratos calculados, por simple proporción directa: $43.2 \mathrm{~mL}$ de solución dextrosa al 50\%. Las indicaciones para el paciente $\mathrm{N}$ quedarán de la siguiente forma:

Dextrosa al 50\% $43.2 \mathrm{~mL}$

$\mathrm{NaCl} 17.7 \%$.................35.9 mL

Agua bidestilada ..........670.9 mL

Total $750.0 \mathrm{~mL}$ para 24 horas

Al trascurrir 24 horas debe obtenerse una nueva determinación de sodio sérico, un nuevo peso y se realizarán los cálculos nuevamente con los datos actuales. No se recomienda la medición de sodio varias veces al día. 
Es conveniente considerar varios puntos:

- Si después de la toma de muestra sanguínea (y antes del resultado de laboratorio) se infundieron líquidos hipoosmolares, el cálculo no será real, porque no se parte de cifras que lo sean. Se requiere que el cálculo se efectúe con una determinación de sodio sérico cercana.

- No deben pasarse cargas rápidas con más de $20 \mathrm{mEq} / \mathrm{L}$ de diferencia por debajo del sodio sérico; en todo caso se considerarán hiposmolares y generará riesgo de edema cerebral y convulsiones. A mayor diferencia en la concentración y mayor volumen infundido, mayor será el riesgo.

- Las soluciones aquí calculadas son hiperosmolares para un paciente en condiciones fisiológicas, pero son las deseables para el estado hiperosmolar del neonato y evitar movimiento masivo de agua.

- El paciente continuará con acidosis metabólica por un tiempo variable; no debe corregirse con bicarbonato, se corregirá paulatinamente conforme avance la rehidratación.

- Entre más sodio sérico tenga el paciente, mayor deberá ser la concentración de sodio en las soluciones; en la elaboración de la mezcla deben usarse ampolletas de cloruro de sodio hipertónico al 17.7\% (contiene $3 \mathrm{mEq}$ de sodio por $\mathrm{mL}$ ) para permitir la infusión de glucosa adecuada (4 a $6 \mathrm{mg} / \mathrm{kg} / \mathrm{min}$ ).

- Si se usa la solución glucosada al 5 o 10\% para llegar al volumen calculado para $24 \mathrm{~h}$ se estará dando una cantidad excesiva de glucosa $/ \mathrm{kg} /$ minuto; lo más conveniente es calcular la glucosa deseada para infusión y administrarla como dextrosa al $50 \%$ y completar el volumen calculado para 24 h con agua bidestilada. Esta última no debe considerarse como el agua libre de la solución y, por supuesto, está contraindicado usar sólo agua bidestilada para diluir el sodio sérico por ser francamente deletérea.

\section{CONCLUSIONES}

No hay una cantidad fija de agua libre que sea útil para todos los grados de deshidratación y concentraciones de sodio sérico, ni tampoco un tiempo de corrección adecuado fijo en todos los casos. El agua libre y, por lo tanto, la concentración de sodio, dependerán del déficit de agua (dado que afecta la proporción de agua corporal) y la concentración actual de sodio sérico; esto es, el tratamiento de la deshidratación hipernatrémica debe ser individualizado.

Datos útiles:

- Cada mEq de un ión ejerce una presión de 1 mOsm; $\mathrm{Na}, \mathrm{K}$ y $\mathrm{Cl}$ son independientes.

- Cada g de glucosa ejerce una presión de 5.04 mOsm

- La solución fisiológica tiene en un litro: $154 \mathrm{mEq} / \mathrm{L}$ de sodio y $154 \mathrm{mEq} / \mathrm{L}$ de cloro; su osmolaridad es de $308 \mathrm{mOsm} / \mathrm{L}$.

- La solución glucosada al 5\% tiene 252 $\mathrm{mOsm} / \mathrm{L}$.

- La solución glucosada al 10\% tiene 504 $\mathrm{mOsm} / \mathrm{L}$.

Hay que recordar que las soluciones con glucosa al 5 o $10 \%$ no participarán de la osmolaridad una vez infundidas porque penetra a la célula con la pérdida de su función osmótica y queda sólo disponible el agua.

Fórmula 4. Osmolaridad sérica $(\mathrm{mOsm} / \mathrm{L})$ :

$$
2(\mathrm{Na}+\mathrm{K})+\frac{\mathrm{BUN}}{2.8}+\frac{\text { Glicemia }}{18}
$$


López Candiani C. Tratamiento de hipernatremia neonatal

\section{REFERENCIAS}

1. Nair S, et al. Clinical profile of neonates with hypernatremic dehydration in an outborn neonatal intensive care unit. Indian Pediatr 2018; 55:343-44. https://doi.org/10.1007/ s13312-018-1268-6

2. Hernández-Alvarez CF, García-Robledo JF, Valdés-López A. Curso clínico de la deshidratación hipernatrémica en recién nacidos. Invest Materno Infant 2014; VI(2): 52-40.

3. Ergenekon $E$, et al. Hypernatremic dehydration in the newborn period and long term follow up. Pediatr Int 2007; 49 (1): 19-23. https://doi.org/10.1111/j.1442200X.2007.02313.x

4. Reilev M, Borch K, Pryds OA. Neonatal hypernatremic dehydration. Why increasing incidence? Ugeskr Laeger 2007; 169 (13): 1227-31.

5. Karadag A, Uras N, Odemis E, Tunc B, Tatli MM. Superior sagital sinus thrombosis: a rare but serious complication of hypernatremic dehydration in newborns. J Perinat Med 2007; 35 (1): 82-3.

6. Hilliard TN, Marsh MJ, Malcom P. Murdoch IA, Wood BP. Radiological case of the month. Arch Pediatr Adolesc Med 1998; 152(11): 1147-1148.

7. Shroff R, Hignett R, Pierce C, Marks S, van't Hoff W. Life-threatening hypernatremic dehydration in breastfed babies. Arch Dis Child 2006; 91: 1025-26.

8. Iglesias Fernández C, Chimenti Camacho P, Vázquez López P, Guerrero Soler M, Blanco Bravo D. Trombosis aórtica y cerebral secundarias a deshidratación hipernatrémica en un recién nacido con lactancia materna exclusiva. An Pediatr (Barc) 2006; 65 (4): 381-3.

9. Ozer PA, Kabatas EU, Kurtul BE, Dilli D, Zenciroglu A, Okumus N. A rare cause of retinal artery occlusion in severe hypernatremic dehydration in newborns. Ophtalmic Surg Lasers Imaging retina 2016; 47(5): 482-485.

10. Han BK, Lee M, Yoon HK. Cranial ultrasound and CT findings in infants with hypernatremic dehydration. Pediatr Radiol 1997; 27: 739-742.

11. Morin C, Chevalier I. Severe hypernatremic dehydration and lower limb gangrene in an infant exposed to lamotrigine, aripiprazole and sertraline in breast milk. Breastfeed Med 2017; 12(6): 377-380.

12. Comay $\mathrm{SC}$, Karabus $\mathrm{CD}$. Peripheral gangrene in hypernatremic dehydration of infancy. Arch Dis Child 1975; 50: 616.

13. Clarke AJ, Sibert JR. Hypernatremic dehydration and necrotizing enterocolitis. Postgrad Med J 1985; 61: 65-66.

14. Chiwane S, Ahmed TM, Bauerfeld CP, Chauhan M. Pulmonary hypertension and right heart failure due to severe hypernatremic dehydration. Perfusion 2017; 32(5): 413-415.

15. Finberg I. Pathogenesis of lesions in the nervous system in hypernatremic states: I. Clinical observations of infants. Pediatrics 1959; 23:40-45.

16. Oddie SJ, Craven V, Deakin K, Westman J, Scally A. Severe neonatal hypernatraemia: a population based study. Arch Dis Child Fetal Neonatal Ed 2013; 98: F384-F387.
17. Richmond S. Hypernatremic dehydration: excess sodium is not the cause. Arch Dis Child Fetal Neonatal Ed 2003; 88(4):F349-F350.

18. Mujawar NS, Jaiswal AN. Hypernatremia in the neonate: Neonatal hypernatremia and hypernatremic dehydration in neonates receiving exclusive breastfeeding. Indian J Crit Care Med 2017; 21(1): 30-33.

19. González García LG, Carrera García L, Arias Llorente RP, Costa Romero M, Suárez Rodríguez M, Ibáñez Fernández A et al. Deshidratación hipernatrémica asociada a la alimentación con lactancia materna en el periodo neonatal. Acta Pediatr Esp 2016; 74(10): 261-265.

20. Boer S, Unal S, van Wouwe JP, van Dommelen P. Evidence based weighing policy during the first week to prevent neonatal hypernatremic dehydration while breastfeeding. PLoS One 2016; 11(12):e0167313.

21. Zaki SA, Mondkar J, Shanbag P, Verma R. Hypernatremic dehydration due to lactation failure in an exclusively breastfed neonate. Saudi J Kidney Dis Transpl 2012; 23(1): 125-128.

22. Boskabadi H, Maamouri G, Ebrahimi M, Ghayour-Mobarhan $\mathrm{M}$, Esmaeily $\mathrm{H}$, Sahebkar $\mathrm{A}$ et al. Neonatal hypernatremia and dehydration in infants receiving inadequate breastfeeding. Asia Pac J Clin Nutr 2010; 19(3): 301-307.

23. Lavagno C, Camozzi P, Renzi S, Lava SAG, Simonetti GD, Bianchetti MG et al. Breastfeeding-associated hipernatremia: A systematic review of the literature. J Human Lactation 2016; 32(1): 67-74.

24. Unal S, Arhan E, Kara N, Uncu N, Aliefendioglu D. Breastfeeding associated hypernatremia: Retrospective analysis of 169 term newborns. Pediatr Int 2008; 50: 29-34.

25. Seri I, Ramanathan R, Evans JR. Acid-Base, fluid and electrolyte management. In: Taeusch HW, Ballard RA, Gleason CA. Avery's diseases of the newborn. 8th ed. Philadelphia: Elsevier Saunders, 2005: 372-397.

26. Borrego Domínguez RR, Imaz Roncero A, López-Herce Cid J, Seriñá Ramírez C. Hipernatremia grave: supervivencia sin secuelas neurológicas. An Pediatr 2003; 58(4): 376-80.

27. Trotman $\mathrm{H}$, Antoine M, Barton M. Hypernatremic dehydration in exclusively breastfed infants: a potentially fatal complication. West Indian Med J 2006; 55 (4): 282-5.

28. Kishaba RG, Losek JD, Eisenberg CSL. Seizure following oral rehydration of hypernatremia with water. Clin Pediatr 1990; 29(4): 239-240.

29. Hogan GR, Dodge PR, Gill SR. Pathogenesis of seizures occurring during restoration of plasma tonicity to normal in animal previously chronically hypernatremic. Pediatrics 1969; 43: 54-64.

30. Ben-Shalom E, Toker O, Schwartz S. Hypernatremic dehydration in Young children: is there a solution?. Isr Med Assoc J 2016; 18(2): 95-99.

31. Rincón DA. Fórmula rápida para la corrección de los trastornos del sodio. Rev Fac Med Univ Nac Colomb 2007; 55(1): 63-65. 
32. López-Candiani C, Salamanca- Galicia O. Hipernatremia en 79 recién nacidos. Factores asociados a desenlace adverso. Acta Pediatr Mex 2012; 33(5): 239-245.

33. Harding D, Moxham J, Cairns P. Weighing alone will not prevent hypernatremic dehydration. Arch Dis Child Fetal Neonatal Ed 2003; 88: F349.

34. Huston RK, Dietz AM, Campbell BB, Dolphin NG, Sklar RS, Wu YK. Enteral water for hypernatremia and intestinal morbidity in infants less than or equal to $1000 \mathrm{~g}$ birth weight.J Perinatol 2007; 27 (1): 32-8.

35. Molteni KH. Initial management of hypernatremic dehydration in the breastfed infant. Clin Pediatr 1994; 33: 731-740.

36. Van Dommelen P, van Wouwe JP, Breuning-Boers JM, van Buuren S, Verkerk PH. Reference chart for relative weight change to detect hypernatraemic dehydration. Arch Dis Child 2007; 92: 490-494. 